Pacific

Journal of

Mathematics

PAYNE-POLYA-WEINBERGER TYPE INEQUALITIES FOR EIGENVALUES OF NONELLIPTIC OPERATORS

Pengcheng Niu and Huiqing Zhang

Volume $208 \quad$ No. 2

February 2003 


\title{
PAYNE-POLYA-WEINBERGER TYPE INEQUALITIES FOR EIGENVALUES OF NONELLIPTIC OPERATORS
}

\author{
Pengcheng Niu and Huiqing Zhang
}

\begin{abstract}
In this paper we consider the eigenvalue problems for some nonelliptic operators which include the real Kohn-Laplacian in the Heisenberg and generalized Baouendi-Grushin operator. Some interest inequalities for eigenvalues are given by establishing the identities and inequalities for noncommutative vector fields.
\end{abstract}

\section{Introduction.}

Let $\triangle$ denote the Laplacian in the Euclidean space. The classic upper estimates, independent of the domain, for the gaps of eigenvalues of $-\triangle,(-\triangle)^{2}$ and $(-\triangle)^{k}(k \geq 3)$ were studied extensively by many mathematicians, cf. Payne, Polya and Weinberger [16], Hile and Yeh [10], Chen and Qian [2], Guo [8] etc.. The asymptotic behaviors of eigenvalues for degenerate elliptic operators were considered by Beals,Greiner and Stanton [1], Menikoff and Sjöstrand [15], Fefferman and Phone [3, 4], Garofalo and Shen [7], respectively.

In this paper, we are concerned with the following eigenvalue problem

$$
\begin{gathered}
-\triangle_{H_{n}} u=\lambda u, \text { in } \Omega, u=0, \text { on } \partial \Omega \\
\left(-\triangle_{H_{n}}\right)^{2} u=\lambda u, \text { in } \Omega, u=\frac{\partial u}{\partial \nu}=0, \text { on } \partial \Omega \\
\left(-\triangle_{H_{n}}\right)^{k} u=\lambda u, \text { in } \Omega, u=\frac{\partial u}{\partial \nu}=\cdots=\frac{\partial^{K-1} u}{\partial \nu^{k-1}}=0, \text { on } \partial \Omega
\end{gathered}
$$

where $\Omega$ is a bounded domain in the Heisenberg group $H_{n}$, with smooth boundary $\partial \Omega, n \geq 1, \nu$ is the unit outward normal to $\partial \Omega, k \geq 3$ is a positive integer. Let $\triangle_{H_{n}}$ denote the real Kohn-Laplacian in the Heisenberg group $\sum_{j=1}^{n}\left(X_{j}^{2}+Y_{j}^{2}\right)$, where $X_{j}=\frac{\partial}{\partial x_{j}}+\frac{y_{j}}{2} \frac{\partial}{\partial t}, Y_{j}=\frac{\partial}{\partial y_{j}}-\frac{x_{j}}{2} \frac{\partial}{\partial t}, j=1, \ldots, n, T=$ $\frac{\partial}{\partial t}$. The existence of eigenvalue for (1.1) has been proved by Luo and Niu $[12,13,14]$ using the Kohn inequality (see [11]) for the vector fields $\left\{X_{j}, Y_{j}\right\}$ together with the spectral properties of compact operators. In what follows we let

$$
0<\lambda_{1} \leq \lambda_{2} \leq \cdots \leq \lambda_{m} \leq \cdots \rightarrow+\infty
$$


denote the successive eigenvalues for (1.1) with corresponding eigenfunctions $u_{1}, u_{2}, \ldots, u_{m}, \ldots$ in $S_{0}^{1,2}(\Omega)$. Here, $S^{1,2}(\Omega)$ denotes the Hilbert space of the functions $u \in L^{2}(\Omega)$ such that $X_{j} u, Y_{j} u \in L^{2}(\Omega)$, and $S_{0}^{1,2}(\Omega)$ denotes closure of $C_{0}^{\infty}(\Omega)$ in the norm

$$
\|u\|_{S^{1,2}}^{2}=\int_{\Omega}\left(\left|\nabla H_{n} u\right|^{2}+|u|^{2}\right) d x d y d t
$$

where $\nabla H_{n} u=\left(x_{1} u, \ldots, X_{n} u, Y-1 u, \ldots, Y_{n} u\right)$ normalized so that

$$
\left\langle u_{i}, u_{j}\right\rangle=\int_{\Omega} u_{i} u_{j} d x d y d t=\delta_{i j}, i, j=1,2 \ldots
$$

For simplicity we will leave out $\Omega$ and $d x d y d t$ in all integrals in the sequel and denote $L=-\triangle_{H_{n}}$.

It is clear that these statements are also valid for the problems (1.2) and (1.3) and the eigenfunctions belong to $S_{0}^{2,2}(\Omega)$ and $S_{0}^{k, 2}(\Omega)$ respectively.

We will derive some upper estimates which are independent of the domain, for the eigenvalues of (1.1), (1.2), and (1.3), respectively. The noncommutativity of vector fields $\left\{X_{j}, Y_{j}\right\}$ makes the discussion of these problems more complicated than one in the case of Euclidean-Laplacian. Furthermore, we will also consider the eigenvalues of generalized Baouendi-Grushin operators [6].

The paper is constructed as follows: Section 2 presents some identities and inequalities based on the vector fields $\left\{x_{j}, Y_{j}, T\right\}, j=1, \ldots, n$, which show the reason that the problems (1.1), (1.2) and (1.3) are treated separately. The main estimates for the eigenvalues of (1.1), (1.2) and (1.3) are given in Section 3, Section 4 and Section 5 respectively. We conclude Section 6 by estimating eigenvalues of the generalized Baouendi-Grushin operator.

\section{Some preliminary lemmas.}

We establish some properties for the commutative vector fields $\left\{x_{j}, Y_{j}, T\right\}$, $(j=1, \ldots, n)$ which are of independent interest.

Lemma 2.1. Given any positive integer $p, 1 \leq p \leq k$, we have

$$
L^{p}\left(\begin{array}{l}
x_{j} u_{i} \\
y_{j} u_{i}
\end{array}\right)=\left(\begin{array}{c}
x_{j} \\
y_{j}
\end{array}\right) L^{p} u_{i}-2\left(\begin{array}{c}
X_{j} \\
Y_{j}
\end{array}\right) L^{p-1} u_{i}-2 \sum_{q=1} p-1 L^{p-q}\left(\begin{array}{c}
X_{j} \\
Y_{j}
\end{array}\right) L^{q-1} u_{i}
$$

$i=1, \ldots, m, \ldots, j=1, \ldots, n$.

Proof. Since

$$
\begin{aligned}
X_{1}\left(x_{1} u_{i}\right) & =u_{i}+x_{1} X_{1} u_{i}, \\
X_{j}\left(x_{1} u_{i}\right) & =x_{1} X_{j} u_{i}, \quad j=2, \ldots, n, \\
Y_{j}\left(x_{1} u_{i}\right) & =x_{1} Y_{j} u_{i}, \quad j=2, \ldots, n,
\end{aligned}
$$


and

$$
\begin{aligned}
X_{1}^{2}\left(x_{1} u_{i}\right) & =2 X_{1} u_{i}+x_{1} X_{1}^{2} u_{i}, \\
X_{j}^{2}\left(x_{1} u_{i}\right) & =x_{1} X_{j}^{2} u_{i}, \quad j=2, \ldots, n, \\
Y_{j}^{2}\left(x_{1} u_{i}\right) & =x_{1} Y_{j}^{2} u_{i}, \quad j=2, \ldots, n,
\end{aligned}
$$

we obtain

$$
L\left(x_{1} u_{i}\right)=x_{1} L u_{i}-2 X_{1} u_{i}
$$

and so

$$
L^{p}\left(x_{1} u_{i}\right)=L^{p-1}\left(x_{1} L u_{i}-2 X_{1} u_{i}\right)=L^{p-1}\left(x_{1} L u_{i}\right)-2 L^{p-1} X_{1} u_{i} .
$$

Changing $u_{i}$ in (2.2) to $L u_{i}$ yields

$$
L\left(x_{1} L u_{i}\right)=x_{1} L^{2} u_{i}-2 X_{1} L u_{i}
$$

and so

$$
L^{p-1}\left(x_{1} L u_{i}\right)=L^{p-2}\left(x_{1} L^{2} u_{i}\right)-2 L^{p-2} X_{1} L u_{i} .
$$

Substituting into (2.3) and repeating these steps, we prove the first formula in (2.1) for $L^{P}\left(x_{1} u_{i}\right)$.

Remark 2.1. When $p=1,2$, we have

$$
\begin{gathered}
L\left(\begin{array}{c}
x_{j} u \\
y_{j} u
\end{array}\right)=\left(\begin{array}{c}
x_{j} \\
y_{j}
\end{array}\right) L u_{i}-2\left(\begin{array}{c}
X_{j} \\
Y_{j}
\end{array}\right) u_{i} \\
L^{2}\left(\begin{array}{c}
x_{j} u_{i} \\
y_{j} u_{i}
\end{array}\right)=\left(\begin{array}{c}
x_{j} \\
y_{j}
\end{array}\right) L^{2} u_{i}-2\left(\begin{array}{c}
X_{j} \\
Y_{j}
\end{array}\right) L u_{i}-2 L\left(\begin{array}{c}
X_{j} \\
Y_{j}
\end{array}\right) u_{i}
\end{gathered}
$$

respectively. It yields the main difference in the following estimations.

Let

$$
0<\lambda_{1} \leq \lambda_{2} \leq \cdots \leq \lambda_{m} \leq \cdots
$$

denote the eigenvalues of (1.1) ((1.2) and (1.3), respectively) with corresponding orthogonal normalized eigenfunctions $u_{1}, u_{2}, \ldots, u_{m}, \ldots$ in $S_{0}^{1,2}(\Omega)$ $\left(S_{0}^{2,2}(\Omega)\right.$ and $S_{0}^{k, 2}(\Omega)$, respectively).

Take the trial functions

$$
\begin{gathered}
\varphi_{i x_{j}}=x_{j} u_{i}-\sum_{i=1}^{m} a_{i l x_{j}} u_{l}, \quad \varphi_{i y_{j}}=y_{j} u_{i}-\sum_{i=1}^{m} a_{i l y_{j}} u_{l} \\
i=1, \ldots, m, j=1, \ldots, n,
\end{gathered}
$$

where $a_{i l x_{j}}=\int x_{j} u_{i} u_{l}, a_{i l y_{j}}=\int y_{i} u_{i} u_{l}$. It is easy to know that each function of (2.6) is orthogonal to $u_{1}, \ldots, u_{m}$ and vanishing on $\partial \Omega$.

Lemma 2.2. For $m \geq 1$, it holds

$$
\sum_{i=1}^{m} \int \varphi_{i x_{j}} X_{j} u_{i}=\sum_{i=1}^{m} \int \varphi_{i y_{j}} Y_{j} u_{i}=-\frac{m}{2}, j=1, \ldots, n \text {. }
$$


Proof. We only prove the first equality in (2.7). Since $a_{i l x_{j}}=a_{l i x_{j}}, \int_{\Omega} u_{l} X_{j} u_{i}$ $=-\int_{\Omega} u_{i} X_{j} u_{l}$ and thus $\sum_{i, l} a_{i l x_{j}} \int u_{l} X_{j} u_{i}=0$, one has

$$
\begin{aligned}
\sum_{i} \int \varphi_{i x_{j}} X_{j} u_{i} & =\sum_{i} \int\left(x_{j} u_{i}-\sum_{i} a_{i l x_{j}} u_{l}\right) X_{j} u_{i} \\
& =\sum_{i} \int x_{j} u_{i} X_{j} u_{i}-\sum_{i} \int X_{j}\left(x_{j} u_{i}\right) u_{i} \\
& =-\sum_{i} \int u_{i}^{2}-\sum_{i} \int x_{j} u_{i} X_{j} u_{i} \\
& =-m-\sum_{i} \int x_{j} u_{i} X_{j} u_{i} .
\end{aligned}
$$

The desired equality is derived immediately.

For simplicity, we let $u_{i} \in C_{0}^{\infty}(\Omega)$ in what follows. Obviously, by the density property, the following results are valid for $u_{i} \in S_{0}^{1,2}(\Omega)$.

Lemma 2.3. For any $p \geq 1$, we have

$$
\left(\int_{\Omega}\left|\nabla_{L}^{p} u_{i}\right|^{2}\right)^{\frac{1}{p}} \leq\left(\int_{\Omega}\left|\nabla_{L}^{p+1} u_{i}\right|^{2}\right)^{\frac{1}{p+1}}, i=1, \ldots, m, \ldots,
$$

where $\nabla_{L} u=\left(X_{1} u, \ldots, X_{n} u, Y_{1} u, \ldots, Y_{n} u\right)$.

Proof. Evidently, for $p \geq 1$

$$
\int\left|\nabla_{L} u_{i}\right|^{2}=-\int u_{i} L u_{i} \leq\left(\int u_{i}^{2}\right)^{\frac{1}{2}}\left(\int\left(L u_{i}\right)^{2}\right)^{\frac{1}{2}}=\left(\int\left|\nabla_{L}^{2} u_{i}\right|^{2}\right)^{\frac{1}{2}}
$$

by the induction assumption it follows that

$$
\begin{aligned}
\int\left|\nabla_{L}^{p} u_{i}\right|^{2} & =-\int \nabla_{L}^{p-1} u_{i} \nabla_{L}^{p+1} u_{i} \\
& \leq\left(\int\left|\nabla_{L}^{p-1} u_{i}\right|^{2}\right)^{\frac{1}{2}}\left(\int\left|\nabla_{L}^{p+1} u_{i}\right|^{2}\right)^{\frac{1}{2}} \\
& \leq\left(\int\left|\nabla_{L}^{p} u_{i}\right|^{2}\right)^{\frac{p-1}{2 p}}\left(\int\left|\nabla_{L}^{p+1} u_{i}\right|^{2}\right)^{\frac{1}{2}} .
\end{aligned}
$$

Therefore the assertion of the Lemma is proved.

As a consequence, we have:

Corollary 2.1. For $k$ in (1.3), we have

$$
\int\left|\nabla_{L} u_{i}\right|^{2} \leq\left(\int\left|\nabla_{L}^{k} u_{i}\right|^{2}\right)^{\frac{1}{k}}
$$


Lemma 2.4. For $p \geq 1$,

$$
L^{p}\left(\begin{array}{c}
X_{j} \\
Y_{j}
\end{array}\right)=\sum_{s=0}^{p} C_{p}^{s}\left(\begin{array}{c}
(-1)^{\left[\frac{s+1}{2}\right]} A_{j}^{s} \\
(-1)^{\left[\frac{s}{2}\right]} A_{j}^{s+1}
\end{array}\right) L^{p-s}(2 T)^{s},
$$

where $j=1, \ldots, n ;[\bullet]$ denote the largest integer part of $\bullet, C_{p}^{s}=\frac{p !}{s !(p-s) 1}$, $A_{j}^{s}=X_{j}$, if $s=0,2,4, \ldots ; A_{j}^{s}=Y_{j}$, if $s=1,3, \ldots$.

Proof. A direct calculation gives

$$
L X_{j}=X_{j} L-2 T Y_{j}, L Y_{j}=Y_{j} L-2 T X_{j}, j-1, \ldots, n .
$$

By the induction assumption that

$$
L^{p-1} X_{j}=\sum_{s=0} p-1 C_{p-1} s(-1)^{\left[\frac{s+1}{2}\right]} A_{j}^{s} L^{p-s-1}(2 T)^{s},
$$

it follows

$$
\begin{aligned}
L^{p} X_{j}= & L\left[\sum_{s=0}^{p-1} C_{p-1} s(-1)^{\left[\frac{s+1}{2}\right]} A_{j}^{s} L^{p-s-1}(2 T)^{s}\right] \\
= & X_{j} L^{P}-2 T Y_{j} L^{p-1} \\
& +\sum_{s=2(s \text { even })}^{p-1} C_{p-1} s(-1)^{\left[\frac{s+1}{2}\right]}\left(X_{j} L-2 T Y_{j}\right) L^{p-s-1}(2 T)^{s} \\
& +\sum_{s=1(s \text { odd })}^{p-1} C_{p-1} s(-1)^{\left[\frac{s+1}{2}\right]}\left(Y_{j} L-2 T X_{j}\right) L^{p-s-1}(2 T)^{s} \\
= & X_{j} L^{P}-2 T Y_{j} L^{p-1} \\
& +\sum_{s=2(s \text { even })}^{p-1} C_{p-1} s(-1)^{\left[\frac{s+1}{2}\right]} X_{j} L^{p-s}(2 T)^{s} \\
& -\sum_{s=2(s \text { even })}^{p-1} C_{p-1} s(-1)^{\left[\frac{s+1}{2}\right]} Y_{j} L^{p-s-1}(2 T)^{s+1} \\
& +\sum_{s=1(s \text { odd })}^{p-1} C_{p-1} s(-1)^{\left[\frac{s+1}{2}\right]} X_{j} L^{p-s}(2 T)^{s} \\
& -\sum_{s=1(s \text { odd })}^{p-1} C_{p-1} s(-1)^{\left[\frac{s+1}{2}\right]} Y_{j} L^{p-s-1}(2 T)^{s+1}
\end{aligned}
$$




$$
\begin{aligned}
= & \sum_{s=0(s \text { even })}^{p-1}\left[C_{p-1} s(-1)^{\left[\frac{s+1}{2}\right]} X_{j} L^{p-s}(2 T)^{s}\right. \\
& \left.+C_{p-1}^{s-1}(-1)^{\left[\frac{s}{2}\right]} X_{j} L^{p-s}(2 T)^{s}\right] \\
& +\sum_{s=1(s \text { odd })}^{p-1}\left[-C_{p-1} s-1(-1)^{\left[\frac{s}{2}\right]} Y_{j} L^{p-s}(2 T)^{s}\right. \\
& \left.+C_{p-1}^{s-1}(-1)^{\left[\frac{s+1}{2}\right]} Y_{j} L^{p-s}(2 T)^{s}\right] \\
= & \sum_{s=0}^{p} C_{p}^{s}(-1)^{\left[\frac{s}{2}\right]} A_{j}^{s} L^{p-s}(2 T)^{s},
\end{aligned}
$$

where we have used that $C_{p-1} s+C_{p-1} s-1=C_{p}^{s}$ in the last equality; $\left[\frac{s+1}{2}\right]=\left[\frac{s}{2}\right]$ if $s$ is even and $\left[\frac{s+1}{2}\right]=\left[\frac{s}{2}\right]+1$ if $s$ is odd. This proves the first equality in (2.10). Another equality is proved similarly.

Lemma 2.5. For $p \geq 1$, it has

$$
\int T L^{p+1} u_{i} \cdot T L^{p} u_{i} \leq \frac{1}{2 n-1} \int L^{p+2} u_{i} \cdot L^{p+1} u_{i}
$$

$$
\int T L^{p} u_{i} \cdot T L^{p} u_{i} \leq \frac{1}{2(2 n-1)}\left[\int L^{p+2} u_{i} \cdot L^{p+1} u_{i}+\int L^{p+1} u_{i} \cdot L^{p} u_{i}\right] .
$$

Proof. Since $t u_{i}=Y_{j} X_{j} u_{i}-X_{j} Y_{j} u_{i}, j=1, \ldots, n$, we get

$$
\begin{aligned}
& 2 n \int T L^{p+1} u_{i} \cdot T L^{p} u_{i} \\
& =2 \int \sum_{j=1}^{n}\left(Y_{j} X_{j}-X_{j} Y_{j}\right) L^{p+1} u_{i} \cdot T L^{p} u_{i} \\
& =2 \sum_{j=1}^{n} \int\left(T X_{j} L^{p} u_{i} \cdot Y_{j} L^{p+1} u_{i}-T Y_{j} L^{p} u_{i} \cdot X_{j} L^{p+1} u_{i}\right) \\
& \leq \sum_{j=1}^{n} \int\left[\left(T X_{j} L^{p} u_{i}\right)^{2}+\left(Y_{j} L^{p+1} u_{i}\right)^{2}+\left(T Y_{j} L^{p} u_{i}\right)^{2}+\left(X_{j} L^{p+1} u_{i}\right)^{2}\right] \\
& =\sum_{j=1}^{n} \int\left[-T X_{j}^{2} L^{p} u_{i} \cdot T L^{p} u_{i}-Y_{j}^{2} L^{p+1} u_{i} \cdot L^{p+1} u_{i}\right. \\
& \left.\quad-T Y_{j}^{2} L^{p} u_{i} \cdot T L^{p} u_{i}-X_{j} 2 L^{p+1} u_{i} \cdot L^{p+1} u_{i}\right] \\
& =\int T L^{p+1} u_{i} \cdot T L^{p} u_{i}+\int L^{p+2} u_{i} \cdot L^{p+1} u_{i}
\end{aligned}
$$


and (2.11) is proved. As for (2.12), one has

$$
\begin{aligned}
2 \int T L^{p} u_{i} \cdot T L^{p} u_{i} & =2 \int T \nabla L L^{p} u_{i} \cdot T \nabla L L^{p-1} u_{i} \\
& \leq \int\left(T \nabla L L^{p} u_{i}\right)^{2}+\int\left(T \nabla L L^{p-1} u_{i}\right)^{2} \\
& =\int T L^{p+1} u_{i} \cdot T L^{p} u_{i}+\int T L^{p} u_{i} \cdot T L^{p-1} u_{i} \\
& \leq \frac{1}{2 n-1}\left[\int L^{p+2} u_{i} \cdot L^{p+1} u_{i}+\int L^{p+1} u_{i} \cdot L^{p} u_{i}\right]
\end{aligned}
$$

and the conclusion is obtained.

Corollary 2.2. For positive integers $a, p \geq 1$, the following inequalities hold:

$$
\int T^{a} L^{p+1} u_{i} \cdot t^{a} L^{p} u_{i} \leq \frac{1}{(2 n-1)^{a}} \int L^{p+a+1} u_{i} \cdot L^{p+a} u_{i}
$$

$$
\int\left(T^{a} L^{p+1} u_{i}\right)^{2} \leq \frac{1}{2(2 n-1)^{a}}\left[\int L^{p+a+1} u_{i} \cdot L^{p+a} u_{i}+L^{p+a} u_{i} \cdot L^{p+a-1} u_{i}\right] .
$$

Proof. It is easy to obtain from (2.11)

$$
\begin{aligned}
\int T^{a} L^{p+1} u_{i} \cdot t^{a} L^{p} u_{i} & \leq \frac{1}{2 n-1} \int T^{a-1} L^{p+2} u_{i} \cdot t^{a-1} L^{p+1} u_{i} \leq \ldots \\
& \leq \frac{1}{(2 n-1)^{a}} \int L^{p+a+1} u_{i} \cdot L^{p+a} u_{i}
\end{aligned}
$$

and from (2.12) and (2.13)

$$
\begin{aligned}
\int\left(T^{a} L^{p} u_{i}\right)^{2} \leq & \frac{1}{2(2 n-1)}\left[\int T^{a-1} L^{p+2} u_{i} \cdot t^{a-1} L^{p+1} u_{i}\right. \\
& \left.\quad+\int T^{a-1} L^{p+1} u_{i} \cdot t^{a-1} L^{p} u_{i}\right] \\
\leq & \frac{1}{2(2 n-1)^{a}}\left[\int L^{p+a+1} u_{i} \cdot L^{p+a} u_{i}+\int L^{p+a} u_{i} \cdot L^{p+a-1} u_{i}\right] .
\end{aligned}
$$

This completes the proof.

\section{Estimates of eigenvalues for (1.1).}

Theorem 3.1. For $m \geq 1$,

$$
\lambda_{m+1}-\lambda_{m} \leq \frac{2}{m n}\left(\sum_{i=1}^{m} \lambda_{i}\right) .
$$


Proof. By the choice of trial function $\varphi_{i x_{1}}$ (see (2.6)) and the Rayleigh-Ritz principle for $\triangle_{H_{n}}$, we have

$$
\lambda_{m+1} \leq \frac{\int\left|\nabla H_{n} \varphi_{i x_{1}}\right|^{2}}{\int\left|\varphi_{i x_{1}}\right|^{2}}, \quad i=1, \ldots, m
$$

and then

$$
\lambda \sum_{i=1}^{m} \int\left|\varphi_{i x_{1}}\right|^{2} \leq \sum_{i=1}^{m} \int\left|\nabla H_{n} \varphi_{i x_{1}}\right|^{2}=\sum_{i=1}^{m} \int L \varphi_{i x_{1}} \cdot \varphi_{i x_{1}} .
$$

By (2.4), the right-hand side becomes $-2 \int \varphi_{i x_{1}} X_{1} u_{i}+\lambda_{i} \int \varphi_{i x_{1}}^{2}$ and we obtain

$$
\left(\lambda_{m+1}-\lambda_{m}\right) \sum_{i=1}^{m} \int \varphi_{i x_{1}}^{2} \leq-2 \sum_{i=1}^{m} \int \varphi_{i x_{1}} X_{1} u_{i} .
$$

Repeating the argument to $\varphi_{i x_{j}}(j=1, \ldots, n)$ yields

$$
\begin{aligned}
& \left(\lambda_{m+1}-\lambda_{m}\right) \sum_{i=1}^{m} \sum_{j=1}^{n} \int\left(\varphi_{i x_{j}}^{2}+\varphi_{i y_{j}}^{2}\right) \\
& \leq-2 \sum_{i=1}^{m} \sum_{j=1}^{n} \int\left(\varphi_{i x_{j}} X_{j} u_{i}+\varphi_{i y_{j}} Y_{j} u_{i}\right) .
\end{aligned}
$$

By Lemma 2.2 and Hölder's inequality

$$
\begin{aligned}
m n= & -\sum_{i=1}^{m} \sum_{j=1}^{n} \int\left(\varphi_{i x_{j}} X_{j} u_{i}+\varphi_{i y_{j}} Y_{j} u_{i}\right) \\
\leq & {\left[\sum_{i, j} \int\left(\varphi_{i x_{j}}^{2}+\varphi_{i y_{j}}^{2}\right)\right]^{\frac{1}{2}}\left[\sum_{i, j} \int\left(X_{j} u_{i}\right)^{2}+\left(Y_{j} u_{i}\right)^{2}\right]^{\frac{1}{2}} } \\
& -\left(\sum_{i} \lambda_{i}\right)^{\frac{1}{2}}\left[\sum_{i, j} \int\left(\varphi_{i x_{j}}^{2}+\varphi_{i y_{j}}^{2}\right)\right]^{\frac{1}{2}}
\end{aligned}
$$

hence we have

$$
\sum_{i, j} \int\left(\varphi_{i x_{j}}^{2}+\varphi_{i y_{j}}^{2}\right) \geq(m n)^{2}\left(\sum_{i} \lambda_{i}\right)^{-1} .
$$

Returning to (3.2), the result is proved.

Remark 3.1. (3.1) is a generalization of Payne-Polya-Weinberger theorem for Dirichlet eigenvalues of Euclidean Laplacian to our context here. 
Introducing a parameter $\alpha>\lambda_{m}$, we have

$$
\begin{aligned}
\left(\lambda_{m+1}-\alpha\right) \sum_{i, j} \int\left(\varphi_{i x_{j}}^{2}+\varphi_{i y_{j}}^{2}\right) \leq & -2 \sum_{i, j} \int\left(\varphi_{i x_{j}} X_{j} u_{i}+\varphi_{i y_{j}} Y_{j} u_{i}\right) \\
& -\sum_{i, j}\left(\alpha-\lambda_{i}\right) \int\left(\varphi_{i x_{j}}^{2}+\varphi_{i y_{j}}^{2}\right)
\end{aligned}
$$

and for some $\delta>0$,

$$
\begin{aligned}
m n= & -\sum_{i, j} \int\left(\varphi_{i x_{j}} X_{j} u_{i}+\varphi_{i y_{j}} Y_{j} u_{i}\right) \\
\leq & \frac{\delta}{2} \sum_{i, j}\left(\alpha-\lambda_{i}\right) \int\left(\varphi_{i x_{j}}^{2}+\varphi_{i y_{j}}^{2}\right) \\
& +\frac{1}{2 \delta} \sum_{i, j}\left(\alpha-\lambda_{i}\right)^{-1} \int\left[\left(X_{j} u_{i}\right)^{2}+\left(Y_{j} u_{i}\right)^{2}\right] .
\end{aligned}
$$

It is easy to see

$$
\left(\lambda_{m+1}-\alpha\right) \sum_{i, j} \int\left(\varphi_{i x_{j}}^{2}+\varphi_{i y_{j}}^{2}\right) \leq 2 m n-m^{2} n^{2}\left(\sum_{i} \frac{\lambda_{i}}{\alpha-\lambda_{i}}\right)^{-1} .
$$

So an extension of Hile-Protter theorem is easily obtained:

Theorem 3.2. For $m \geq 1$, one has

$$
\sum_{i=1} m \frac{\lambda_{i}}{\lambda_{m+1}-\lambda_{i}} \geq \frac{m n}{2}
$$

\section{Estimates of eigenvalues for (1.2).}

We denote the eigenvalues of (1.2) by

$$
0<\lambda_{1} \leq \lambda_{2} \leq \cdots \leq \lambda_{m} \leq \cdots \rightarrow \infty
$$

with corresponding eigenfunctions $u_{1}, u_{2}, \ldots, u_{m}, \ldots$ in $S_{0}^{2,2}(\Omega)$.

Theorem 4.1. Let $m \geq 1$. then the following estimate holds

$$
\lambda_{m+1}-\lambda_{m} \leq \frac{4(n+1)}{m^{2} n^{2}}\left(\sum_{i=1}^{m} \sqrt{\lambda_{i}}\right)^{\frac{1}{2}} .
$$

Proof. Following the argument of (3.3) and noting (2.5), we have

$$
\left(\lambda_{m+1}-\alpha\right) S \leq J-T,
$$


where $\alpha$ is a parameter, $\alpha>\lambda_{m}$,

$$
\begin{aligned}
S & =\sum_{i, j} \int\left(\varphi_{i x_{j}}^{2}+\varphi_{i y_{j}}^{2}\right), \\
J & =-2 \sum_{i, j} \int\left[\left(L X_{j}+X_{j} L\right) u_{i} \cdot \varphi_{i x_{j}}+\left(L Y_{j}+Y_{j} L\right) u_{i} \cdot \varphi_{i y_{j}}\right], \\
T & =\sum_{i, j}\left(\alpha-\lambda_{j}\right) \int\left(\varphi_{i x_{j}}^{2}+\varphi_{i y_{j}}^{2}\right) .
\end{aligned}
$$

We need two propositions:

\section{Proposition 4.1.}

$$
J \leq 4(n+1) \sum_{i=1}^{m} \sqrt{\lambda_{i}} .
$$

Proof. Noting (2.6), one has

$$
\begin{aligned}
& \sum_{i} \int\left(L X_{j}+X_{j} L\right) u_{i} \cdot \varphi_{i x_{j}} \\
& =\sum_{i} \int\left(L X_{j}+X_{j} L\right) u_{i}\left(x_{j} u_{i}-\sum_{l} a_{i l x_{j}} u_{l}\right) \\
& =\sum_{i} \int\left(X_{j} u_{i} L\left(x_{j} u_{i}\right)-L u_{i} \cdot X_{j}\left(x_{j} u_{i}\right)\right) \\
& \quad-\sum_{i, l} \int\left(a_{i l x_{j}} X_{j} u_{i} L u_{l}-a_{i l x_{j}} L u_{i} \cdot X_{j} u_{l}\right) \\
& \triangleq M_{1}+M_{2} .
\end{aligned}
$$

Since $a_{i l x_{j}}=a_{l i x_{j}}, i, l=1, \ldots, m$, we see $M_{2}=0$. On the other hand, (2.4) implies

$$
\begin{aligned}
& \sum_{i} \int\left(L X_{j}+X_{j} L\right) u_{i} \cdot \varphi_{i x_{j}} \\
& =M_{1}=\sum_{i} \int\left[X_{j} u_{i}\left(-2 X_{j} u_{i}+x_{j} L u_{i}\right)-L u_{i}\left(u_{i}+x_{j} X_{j} u_{i}\right)\right] \\
& =\sum_{i} \int\left(2 X_{j}^{2} u_{i} \cdot u_{i}-u_{i} L u_{i}\right) .
\end{aligned}
$$

Similarly, we have

$$
\sum_{i} \int\left(L Y_{j}+Y_{j} L\right) u_{i} \cdot \varphi_{i y_{j}}=\sum_{i} \int\left(2 Y_{j}^{2} u_{i}-u_{i} L u_{i}\right) .
$$


Therefore by Hölder's inequality we obtain

$$
\begin{aligned}
J & =-2 \sum_{i}\left[\int-2 L u_{i} \cdot u_{i}-2 n \int u_{i} L u_{i}\right] \\
& =4(n+1) \sum_{i} \int u_{i} L U_{i} \\
& \leq 4(n+1) \sum_{i}\left(\int u_{i}^{2}\right)^{\frac{1}{2}}\left(\int\left(L u_{i}\right)^{2}\right)^{\frac{1}{2}} \\
& =4(n+1) \sum_{i} \sqrt{\lambda_{i}} .
\end{aligned}
$$

\section{Proposition 4.2.}

$$
T \geq m^{2} n^{2}\left(\sum_{i} \frac{\sqrt{\lambda_{i}}}{\alpha-\lambda_{i}}\right)^{-1}
$$

Proof. By Lemma 2.2 and Hölder's inequality we have

$$
\begin{aligned}
m n & =-\sum_{i, j} \int\left(\varphi_{i x_{j}} X_{j} u_{i}+\varphi_{i y_{i}} Y_{j} u_{i}\right) \\
& \leq \sum_{i}\left[\sum_{j} \int\left(\varphi_{i x_{j}}^{2}+\varphi_{i y_{j}}^{2}\right)\right]^{\frac{1}{2}}\left[\sum_{i} \int\left(\left(X_{j} u_{i}\right)^{2}+\left(Y_{j} u_{i}\right)^{2}\right)\right]^{\frac{1}{2}} \\
& \leq \frac{\delta}{2} \sum_{i, j}\left(\alpha-\lambda_{i}\right) \int\left(\varphi_{i x_{j}}^{2}+\varphi_{i y_{j}}^{2}\right)+\frac{1}{2 \delta} \sum_{i} \frac{1}{\alpha-\lambda_{i}} \int u_{i} L u_{i} \\
& \leq \frac{\delta}{2} T+\frac{1}{2 \delta} \sum_{i} \frac{\sqrt{\lambda_{i}}}{\alpha-\lambda_{i}}
\end{aligned}
$$

where $\delta$ is some positive parameter. After minimizing the right-hand side of (4.5), the result is proved.

Proof of Theorem 4.1. Substituting (4.3) and (4.4) into (4.2) yields

$$
\left(\lambda_{m+1}-\alpha\right) S \leq 4(n+1) \sum_{i} \sqrt{\lambda_{i}}-m^{2} n^{2}\left(\sum_{i} \frac{\sqrt{\lambda_{i}}}{\alpha-\lambda_{i}}\right)^{-1} .
$$

The inequality (4.1) is obtained with the similar discussion in [10]. 


\section{Estimates of eigenvalues for (1.3).}

Theorem 5.1. If $k \geq 3$ is odd, then

$$
\lambda_{m+1}-\lambda_{m} \leq \frac{\sum_{i=1}^{m} \lambda_{i}^{\frac{1}{k}}}{m^{2} n^{2}}\left[(2 n+4) k \sum_{i=1}^{m} \lambda_{i}^{\frac{k-1}{k}}+C_{1}(n, k) \sum_{i=1}^{m}\left(\lambda_{i}+\lambda_{i}^{\frac{k-2}{k}}\right)\right]
$$

and if $k \geq 4$ is even, then

$$
\lambda_{m+1}-\lambda_{m} \leq \frac{\sum_{i=1}^{m} \lambda_{i}^{\frac{1}{k}}}{m^{2} n^{2}}\left[(2 n+4) k \sum_{i=1}^{m} \lambda_{i}^{\frac{k-1}{k}}+C_{2}(n, k) \sum_{i=1}^{m} \lambda_{i}^{\frac{k-1}{k}}\right],
$$

where $C_{1}(n, k)$ and $C_{2}(n, k)$ are the constants depending on $n$ and $k$.

Proof. Using the trial function $\varphi_{i x_{1}}$ (see (2.6)) and the Rayleigh-Ritz inequality, we have

$$
\begin{aligned}
\lambda_{m+1} & \leq \int \varphi_{i x_{1}} L^{K} \varphi_{i x_{1}} \\
& =\int \varphi_{i x_{1}} L^{k}\left(x_{1} u_{i}-\sum_{l=1}^{m} a_{i l x_{1}} u_{l}\right) \\
& =\int \varphi_{i x_{1}}\left(\lambda_{i} x_{1} u_{i}-2 \sum_{q=1}^{k} L^{k-q} X_{1} L^{q-1} u_{i}\right) \\
& =\lambda_{i} \int \varphi_{i x_{1}}^{2}-2 \int\left(\sum_{q=1}^{k} L^{k-q} X_{1} L^{q}-1 u_{i}\right) \varphi_{i x_{1}}, i=1, \ldots, m .
\end{aligned}
$$

Introducing a parameter $\beta$, we have

$$
\left(\lambda_{m+1}-\beta\right) \sum_{i} \int \varphi_{i x_{1}}^{2} \leq \sum_{i}\left(\alpha_{i}-\beta\right) \int \varphi_{i x_{1}}^{2}-2 \sum_{i, q} \int L^{k-q} X_{1} L^{q-1} u_{i} \cdot \varphi_{i x_{1}} .
$$

Let

$$
\begin{aligned}
& S=\sum_{i=1}^{m} \sum_{j=1}^{n} \int\left(\varphi_{i x_{j}}^{2}+\varphi_{i y_{j}}^{2}\right), \\
& I_{1}=\sum_{i=1}^{m} \sum_{j=1}^{n}\left(\beta-\lambda_{j}\right) \int\left(\varphi_{i x_{j}}^{2}+\varphi_{i y_{j}}^{2}\right), \\
& I_{2}=-2 \sum_{i=1}^{m} \sum_{j=1}^{n} \sum_{q-1}^{k} \int\left(\varphi_{i x_{j}} L^{k-q} X_{j} L^{q-1} u_{i}+\varphi_{i y_{j}} L^{k-q} X_{j} L^{q-1} u_{i}\right),
\end{aligned}
$$


we have

$$
\left(\lambda_{m+1}-\beta\right) S+I_{1} \leq I_{2} .
$$

Applying Lemma 2.2 yields

$$
m \leq\left[\sum_{i=1}^{m} \int\left(\varphi_{i x_{j}}^{2}+\varphi_{i y_{j}}^{2}\right)\right]^{\frac{1}{2}}\left[\sum_{i=1}^{m} \int\left[\left(X_{j} u_{i}\right)^{2}+\left(Y_{j} u_{i}\right)^{2}\right]\right]^{\frac{1}{2}}
$$

and summing over $j$ gives

$$
2 m n \leq \delta I_{1}+\frac{1}{\delta} \sum_{i=1}^{m} \frac{1}{\beta-\lambda_{i}} \int\left|\nabla_{L} u_{i}\right|^{2}
$$

where $\delta$ is a positive number. Then we have by Corollary 2.1

$$
2 m n \leq \delta I_{1}+\frac{1}{\delta}\left(\sum_{i=1}^{m} \frac{\lambda_{i}^{\frac{1}{k}}}{\beta-\lambda_{i}}\right)
$$

After choosing the minimum of the right-hand side, we derive a lower bound of $I_{1}$ :

$$
I_{1} \geq m^{2} n^{2}\left(\sum_{i=1}^{m} \frac{\lambda_{i}^{\frac{1}{k}}}{\beta-\lambda_{i}}\right)^{-1}
$$

Now we estimate $I_{2}$. Notice the following relation by $(2.1)$

$-\sum_{i, l, q} a_{i l x_{j}} \int u_{l} L^{q-1} X_{j} L^{q-1} u_{i}=\frac{1}{2} \sum_{i, L} a_{i l x_{j}} \int\left(u_{l} L^{k}\left(x_{j} u_{i}\right)-x_{j} u_{l} L^{k} u_{i}\right)=0$

and

$$
-\sum_{i, l, q} a_{i l y_{j}} \int u_{l} L^{q-1} Y_{j} L^{q-1} u_{i}=0, \quad i, l=1, \ldots, m, q=1, \ldots, k .
$$

Therefore we have

$$
\begin{aligned}
I_{2}= & -2 \sum_{i, j, q} \int\left(x_{j} u_{i} L^{k-q} X_{j} L^{q-1} u_{i}+y_{j} u_{i} L^{k-q} Y_{j} L^{q-1} u_{i}\right) \\
= & -2 \sum_{i, j, q} \int\left(L^{k-q}\left(x_{j} u_{i}\right) X_{j} L^{q-1} u_{i}+L^{k-q}\left(y_{j} u_{i}\right) Y_{J} L^{q-1} U_{i}\right) \\
= & -2 \sum_{i, j, q}\left\{\int \left(\left(x_{j} L^{k-q} u_{i}-2 \sum_{r=1}^{k-q-1} L^{k-q-r} X_{j} L^{r-1} u_{i}\right) X_{j} L^{q-1} u_{i}\right.\right. \\
& \left.\quad-2 X_{j} L^{k-q-1} u_{i} \cdot X_{j} L^{q-1} u_{i}\right)
\end{aligned}
$$




$$
\left.+\int\left(y_{j} L^{k-q} u_{i}-2 \sum_{r=1}^{k-q-1} L^{k-q-r} Y_{j} L^{q-1} u_{i}-2 Y_{j} L^{k-q-1} u_{i} \cdot Y_{j} L^{q-1} u_{i}\right)\right\} .
$$

Since

$$
\begin{aligned}
& \sum_{q=1}^{k} \int\left(\begin{array}{c}
x_{j} \\
y_{j}
\end{array}\right) L^{k-q} u_{i} \cdot\left(\begin{array}{c}
X_{j} \\
Y_{j}
\end{array}\right) L^{q-1} u_{i} \\
& =-\sum_{q-1}^{k}\left[\int L^{k-q} u_{i} \cdot L_{q-1} u_{i}+\left(\begin{array}{c}
x_{j} X_{j} \\
y_{j} Y_{j}
\end{array}\right) L^{k-q} u_{i} \cdot L^{q-1} u_{i}\right]
\end{aligned}
$$

we have

$$
\begin{aligned}
& \sum_{q=1}^{k} \int\left(\begin{array}{c}
x_{j} \\
y_{j}
\end{array}\right) L^{k-q} u_{i} \cdot\left(\begin{array}{c}
X_{j} \\
Y_{j}
\end{array}\right) L^{q-1} u_{i} \\
& =-\frac{1}{2} \sum_{q-1}^{k} \int L^{k-q} u_{i} \cdot L_{q-1} u_{i} \\
& =-\frac{k}{2} \int L^{k-1} u_{i} \cdot u_{i} .
\end{aligned}
$$

On the other hand

$$
\begin{aligned}
& \sum_{j} \int\left(X_{j} L^{k-q-1} U_{i} \cdot X_{j} L^{q-1} U_{i}+Y_{j} L^{k-q-1} u_{i} \cdot Y_{j} L^{q-1} u_{i}\right) \\
& =\int L^{k-1} u_{i} \cdot u_{i}
\end{aligned}
$$

so it follows

(5.6)

$$
\begin{aligned}
I_{2}= & (2 n+4) k \sum_{i=1}^{m} \int L^{k-1} u_{i} \cdot u_{i} \\
& +4 \sum_{i, j, q} \int\left(\sum_{r=1}^{k-q-1} L^{k-q-r} X_{j} L^{r-1} u_{i} \cdot X_{j} L^{q-1} u_{i}\right. \\
& \left.+\sum_{r=1}^{k-q-1} L^{k-q-r} Y_{j} L^{r-1} u_{i} \cdot Y_{j} L^{q-1} u_{i}\right) \\
= & (2 n+4) k \sum_{i=1}^{m} \int L^{k-1} u_{i} \cdot u_{i} \\
& +4 \sum_{i, j, q} \sum_{r=1}^{k-q-r}\left[\sum_{s=0}^{k-q-r} C_{k-r-q}^{s}(-1)^{\left[\frac{s+1}{2}\right]} A_{j}^{s}\right. \\
& \cdot L^{k-q-r-s}(2 T)^{s} L^{r-1} u_{i} \cdot X_{j} L^{q-1} u_{i}
\end{aligned}
$$




$$
\begin{array}{r}
\left.+\sum_{s=0}^{k-q-r} C_{k-q-r}^{s}(-1)^{\left[\frac{s}{2}\right]} A_{j}^{s+1} L^{k-q-r-s}(2 T)^{s} L^{r-1} u_{i} \cdot Y_{j} L^{q-1} u_{i}\right] \\
\triangleq(2 n+4) k \sum_{i=1}^{m} \int L^{k-1} u_{i} \cdot u_{i}+4 \sum_{i, j, q} \sum_{r=1}^{k-q-1}\left(I_{3}+I_{4}\right)
\end{array}
$$

where we have used Lemma 2.4. We obtain that

$$
\begin{aligned}
& I_{3(s \text { odd })}+I_{4(\text { s odd })} \\
& =\sum_{s \leq k-q-r, s \text { odd }}\left[\int C_{k-q-r}^{s}\left(-(-1)^{\left[\frac{s}{2}\right]}\right) Y_{j} L^{k-q-r-s}(2 T)^{s} L^{r-1} u_{i} \cdot X_{j} L^{q-1} u_{i}\right. \\
& \left.+\int C_{k-q-r}^{s}(-1)^{\left[\frac{s}{2}\right]} X_{j} L^{k-q-r-s}(2 T)^{s} L^{r-1} u_{i} \cdot Y_{j} L^{q-1} u_{i}\right] \\
& =-\sum_{s \leq k-q-r, s \text { odd }} \int C_{k-q-r}^{s}(-1)^{\left[\frac{s}{2}\right]}\left(Y_{J} X_{j}-X_{j} Y_{j}\right) \\
& \cdot L^{k-q-r-s}(2 T)^{s} L^{r-1} u_{i} \cdot L^{q-1} u_{i} \\
& =-\sum_{s \leq k-q-r, s \text { odd }} 2^{s} C_{k-q-r}^{s}(-1)^{\left[\frac{s}{2}\right]} \int T^{s+1} L^{k-q-s-1} u_{i} \cdot L^{q-1} u_{i} \\
& =-\sum_{s \leq k-q-r, s \text { odd }} 2^{s} C_{k-q-r}^{s}(-1)^{\left[\frac{s}{2}\right]} \int T^{s+1} u_{i} \cdot L^{k-s-2} u_{i}
\end{aligned}
$$

where $T=Y_{j} X_{j}-X_{j} Y_{j}$. Similarly,

$$
I_{3(s \text { even })}+I_{4(\text { s even })}=\sum_{s \leq k-q-r, s \text { even }} 2^{s} C_{k-q-r}^{s}(-1)^{\left[\frac{s}{2}\right]} \int T^{s} u_{i} \cdot L^{k-s-1} u_{i} .
$$

First let $k$ be odd. If $s$ is odd, then by (2.14)

$$
\begin{aligned}
& (-1)^{1+\left[\frac{s}{2}\right]} \int T^{s+1} u_{i} \cdot L^{k-s-2} u_{i} \\
& =(-1)^{\frac{s+1}{2}+\left[\frac{s}{2}\right]+1} \int\left(T^{\frac{s+1}{2}} L^{\frac{k-s-2}{2}} u_{i}\right)^{2} \\
& \leq \frac{1}{2(2 n-1)^{\frac{s+1}{2}}}\left[\int L^{\frac{k-1}{2}+1} u_{i} \cdot L^{\frac{k-1}{2}} u_{i}+\int L^{\frac{k-1}{2}} u_{i} \cdot L^{\frac{k-1}{2}-1} u_{i}\right] \\
& =\frac{1}{2(2 n-1)^{\frac{s+1}{2}}}\left[\int L^{k} u_{i} \cdot u_{i}+\int L^{k-2} u_{i} \cdot u_{i}\right]
\end{aligned}
$$


and if $s$ is even, then

$$
\begin{aligned}
& (-1)^{\left[\frac{s}{2}\right]} \int T^{s} u_{i} \cdot L^{k-s-1} u_{i} \\
& =(-1)^{\frac{s}{2}+\left[\frac{s}{2}\right]} \int\left(T^{\frac{s}{2}} L^{\frac{k-s-1}{2}} u_{i}\right)^{2} \\
& \leq \frac{1}{2(2 n-1)^{\frac{s}{2}}}\left[\int L^{\frac{k-1}{2}+1} u_{i} \cdot L^{\frac{k-1}{2}} u_{i}+\int L^{\frac{k-1}{2}} u_{i} \cdot L^{\frac{k-1}{2}-1} u_{i}\right] \\
& =\frac{1}{2(2 n-1)^{\frac{s}{2}}}\left[\int L^{k} u_{i} \cdot u_{i}+\int L^{k-2} u_{i} \cdot u_{i}\right](k \geq 3) .
\end{aligned}
$$

Summing (5.9) and (5.10) yields

$$
\begin{aligned}
I_{2} \leq & (2 n+4) k \sum_{i=1}^{m} \int L^{k-1} u_{i} \cdot u_{i} \\
& +4 \sum_{i, j, q} \sum_{r=1}^{k-q-1}\left[\sum_{s \leq k-q-r(s \text { odd })} 2^{s} C_{k-q-r}^{s} \frac{1}{2(2 n-1)^{\frac{s+1}{2}}}\right. \\
& \cdot\left(\int L^{k} u_{i} \cdot u_{i}+\int L^{k-2} u_{i} \cdot u_{i}\right) \\
& \left.+\sum_{s \leq k-q-r(s \text { even })} 2^{s} C_{k-q-r}^{s} \frac{1}{2(2 n-1)^{\frac{s}{2}}}\left(\int L^{k} u_{i} \cdot u_{i}+\int L^{k-2} u_{i} \cdot u_{i}\right)\right] \\
\leq & (2 n+4) k \sum_{i=1}^{m} \int L^{k-1} u_{i} \cdot u_{i} \\
= & 2 \sum_{i, j, q} \sum_{r=1}^{k-q-1}\left(\sum_{s \leq k-q-r(s \text { odd })} \frac{2^{s} C_{k-q-r}^{s}}{(2 n-1)^{\frac{s+1}{2}}}+\sum_{s \leq k-q-r(s \text { even })} \frac{2^{s} C_{k-q-r}^{s}}{(2 n-1)^{\frac{s}{2}}}\right) \\
& \cdot\left(\int^{k} u_{i} \cdot u_{i}+\int L^{k-2} u_{i} \cdot u_{i}\right) \\
\leq & (2 n+4) k \sum_{i=1}^{m}\left(\int L^{k} u_{i} \cdot u_{i}\right)^{\frac{k-1}{k}} \\
& +C_{1}(n, k) \sum_{i=1}^{m}\left[\lambda_{i}+\left(\int L^{k} u_{i} \cdot u_{i}\right)^{\frac{k-2}{k}}\right] \\
& +4) k \sum_{i=1}^{m} \lambda_{i}^{\frac{k-1}{k}}+C_{1}(n, k) \sum_{i=1}^{m}\left[\lambda_{i}+\lambda_{i}^{\frac{k-2}{k}}\right],
\end{aligned}
$$


where we have used Lemma 2.3. Note that

$$
C_{1}(n, k) \leq 2 n \sum_{q=1}^{k} \sum_{r=1}^{k-q-1}\left(1+\frac{2}{(2 n-1)^{\frac{1}{2}}}\right)^{k-q-r}
$$

Substituting (5.11) in (5.3) leads to

$$
\begin{aligned}
\left(\lambda_{m+1}-\beta\right) s \leq & (2 n+4) k \sum_{i=1}^{m} \lambda_{i}^{\frac{k-1}{k}} \\
& +C_{1}(n, k) \sum_{i=1}^{m}\left(\lambda_{i}+\lambda_{i}^{\frac{k-1}{k}}\right)-m^{2} n^{2}\left(\sum_{i=1}^{m} \frac{\lambda_{i}^{\frac{1}{k}}}{\beta-\lambda_{i}}\right)^{-1} .
\end{aligned}
$$

Along with the method in [10], we obtained (5.1).

Now let $k$ be even. If $s$ is odd, then by Corollary 2.2

$$
\begin{aligned}
(-1)^{\left[\frac{s}{2}\right]+1} \int T^{s+1} u_{i} L^{k-s-2} u_{i} & =\int T^{\frac{s+1}{2}} L^{\frac{k-s-3}{2}} u_{i} \cdot T^{\frac{s+1}{2}} L^{\frac{k-s-3}{2}+1} u_{i} \\
& \leq \frac{1}{(2 n-1)^{\frac{s+1}{2}}} \int L^{\frac{k-2}{2}+1} u_{i} L^{\frac{k-2}{2}} u_{i} \\
& =\frac{1}{(2 n-1)^{\frac{s+1}{2}}} \int L^{k-1} u_{i} \cdot u_{i}
\end{aligned}
$$

and if $s$ is even, then

$$
\begin{aligned}
(-1)^{\left[\frac{s}{2}\right]} \int T^{s} u_{i} L^{k-s-1} u_{i} & =\int T^{\frac{s}{2}} L^{\frac{k-s-2}{2}} u_{i} \cdot T^{\frac{s}{2}} L^{\frac{k-s-2}{2}+1} u_{i} \\
& \leq \frac{1}{(2 n-1)^{\frac{s}{2}}} \int L^{\frac{k-2}{2}} u_{i} L^{\frac{k-2}{2}+1} u_{i} \\
& =\frac{1}{(2 n-1)^{\frac{s}{2}}} \int L^{k-1} u_{i} \cdot u_{i} .
\end{aligned}
$$


Summing two inequalities above yields

$$
\begin{aligned}
& I_{2}^{\prime} \leq(2 n+4) K \sum_{i=1}^{m} \int L^{k-1} u_{i} \cdot u_{i} \\
&+4 \sum_{i, j, q} \sum_{r=1}^{k-q-1}\left(\sum_{s \leq k-q-r(s \text { odd })} \frac{2^{s} C_{k-q-r}^{s}}{(2 n-1)^{\frac{s+1}{2}}}\right. \\
&\left.+\sum_{s \leq k-q-r(s \text { even })} \frac{2^{s} C_{k-q-r}^{s}}{(2 n-1)^{\frac{s}{2}}}\right) \int L^{k-1} u_{i} \cdot u_{i} \\
& \triangleq(2 n+4) k \sum_{i=1}^{m} \int L^{k-1} u_{i} \cdot u_{i}+C_{2}(n, k) \sum_{i=1}^{m} \int L^{k-1} u_{i} \cdot u_{i} \\
& \leq(2 n+4) k \sum_{i=1}^{m} \lambda_{i}^{\frac{k-1}{k}}+C_{2}(n, k) \sum_{i=1}^{m} \lambda_{i}^{\frac{k-1}{k}} .
\end{aligned}
$$

Note that

$$
C_{2}(n, k) \leq 4 n \sum_{q=1}^{k} \sum_{r=1}^{k-q-1}\left(1+\frac{2}{(2 n-1)^{\frac{1}{2}}}\right)^{k-q-r} .
$$

Then proceeding with the same way for proving (5.1) we deduce (5.2).

\section{Eigenvalues for generalized Baouendi-Grushin operator.}

Consider the eigenvalue problem

$$
\begin{cases}-L u=\lambda u, & \text { in } \Omega \\ u=0 & \text { on } \Omega\end{cases}
$$

where $L$ denote the generalized Baouendi-Grushin operator

$$
L=\sum_{i=1}^{M} X_{i}^{2}+\sum_{j=1}^{N} Y_{j}^{2}
$$

with the nonsmooth vector fields

$$
X_{j}=\frac{\partial}{\partial x_{i}}, \quad i=1, \ldots, M, \quad Y_{j}=|x|^{\alpha} \frac{\partial}{\partial y_{j}}, \quad j=1, \ldots, N,
$$

$\alpha \geq 1, \alpha \in R, M, N \geq 1$ and $M+N=n$. $\Omega$ is a bounded domain in $R^{n}$ with smooth boundary $\partial \Omega$.

The existence of eigenvalues for the problem (6.1) can be proved with the method in [12] and the embedding theorem in [6] and then (6.1) possesses a system of eigenfunctions $\left\{u_{h}\right\}$ that forms an orthonormal base, with the corresponding eigenvalues $\left\{\lambda_{h}\right\}: 0<\lambda_{1} \leq \lambda_{2} \leq \ldots$. 
By choosing the trial functions

(6.4) $\varphi_{h x_{i}}=x_{i} u_{h}-\sum_{i=1}^{m} a_{h l x_{j}} u_{l}, \quad \varphi_{h y_{i}}=y_{i} u_{h}-\sum_{i=1}^{m} a_{h l y_{j}} u_{l}, h=1, \ldots, m$, we have

$$
\begin{aligned}
& \left(\lambda_{m+1}-\lambda_{m}\right) \sum_{h=1}^{m}\left(\sum_{i=1}^{M} \int \varphi_{h x_{i}}^{2}+\sum_{j=1}^{N} \int \varphi_{h y_{j}}^{2}\right) \\
& \leq-2 \sum_{l=1}^{m}\left(\sum_{i=1}^{M} \varphi_{h x_{i}} X_{i} u_{h}+\sum_{j=1}^{N} \int|x|^{\alpha} \varphi_{h y_{j}} Y_{i} u_{h}\right) .
\end{aligned}
$$

Noting that

$$
\begin{aligned}
\sum_{i=1}^{M} \sum_{h=1}^{m} \int \varphi_{h x_{i}} X_{i} u_{h} & =-\frac{m M}{2}, \\
\sum_{j=1}^{N} \sum_{h=1}^{m} \int \varphi_{h y_{j}} Y_{i} u_{h} & =-\frac{N}{2} \sum_{h=1}^{m} \int|x|^{2 \alpha} u_{h}^{2}
\end{aligned}
$$

and Schwarz's inequality, we get

$$
\begin{aligned}
& \frac{m M}{2} \leq \frac{m M}{2}+\frac{N}{2} \sum_{h=1}^{m} \int|x|^{2 \alpha} u_{h}^{2} \\
& \leq \sum_{h=1}^{m}\left[\sum_{i=1}^{M} \int\left|\varphi_{h x_{i}} X_{i} u_{h}\right|+\sum_{j=1}^{N} \int|x|^{\alpha}\left|\varphi_{h y_{j}} Y_{j} u_{h}\right|\right] \\
& \leq \max \left(1, d^{\alpha}\right)\left[\sum_{h=1}^{m}\left(\sum_{i=1}^{M} \int\left|\varphi_{h x_{i}}\right|^{2}+\sum_{j=1}^{N} \int\left|\varphi_{h y_{i}}\right|^{2}\right)\right]^{\frac{1}{2}} \\
& \cdot\left[\sum_{h=1}^{m} \int\left(-L u_{h}\right) u_{h}\right]^{\frac{1}{2}}
\end{aligned}
$$

where $d$ is the diameter of $\Omega_{y}$, the projection of $\Omega$ in the $y$-space. Then

$$
\sum_{h=1}^{m}\left(\sum_{i=1}^{M} \int\left|\varphi_{h x_{i}}\right|^{2}+\sum_{j=1}^{N} \int\left|\varphi_{h y_{j}}\right|^{2}\right) \geq \frac{m^{2} M^{2}}{4 \max \left(1, d^{2 \alpha}\right) \sum_{h=1}^{m} \lambda_{h}} .
$$

Returning to (6.5), we prove the following: 
Theorem 6.1. Let $m \geq 1$, then

$$
\lambda_{m+1}=\lambda_{m} \leq \frac{4 n}{m M^{2}} \max \left(1, d^{4 \alpha}\right) \sum_{h=1}^{m} \lambda_{h} .
$$

Remark 6.1. This shows that the upper bounds for eigenvalues of (6.1) depends on the domain.

Acknowledgments. This research was supported by the National Natural Science Foundation of China. The authors would like to thank Professor Luo Xuebo for his hospitality. They also would like to thank the referees for helpful comments and suggestions.

\section{References}

[1] R. Beals, P.C. Greiner and N.K. Stanton, The heat equation on a CR manifold, J. Diff. Geom., 20 (1984), 343-387, MR 86g:58135, Zbl 0553.58029.

[2] Z. Chen and C. Qian, On the upper bound of eigenvalues for elliptic equation with higher orders, J. Math. Anal. Appl., 186 (1994), 821-834, MR 95f:35181, Zbl 0814.35082.

[3] C. Fefferman and D.H. Phong, Subelliptic eigenvalue problems, Conf. in Honor of A. Zygmund, Wadsworth Math. Series, 1981, MR 86c:35112, Zbl 0503.35071.

[4] - On the lowest eigenvalue of a pseudodifferential operator, Proc. Nat. Acad. Sci., 76 (1979), 6055-6056, MR 81d:47032, Zbl 0434.35071.

[5] G. Folland and E.M. Stein, Estimates for the $\bar{\partial}_{b}$ complex and analysis on the Heisenberg group, Comm. Pure Appl. Math., 27 (1974), 429-522, MR 51 \#3719, Zbl 0293.35012.

[6] N. Garofalo and D. Nhieu, Isoperimetric and Sobolev inequalities for CarnotCarathéodory spaces and the existence of minimal surfaces, Comm. Pure Appl. Math., 49 (1996), 1081-1144, MR 97i:58032, Zbl 0880.35032.

[7] N. Garofalo and Z. Shen, Absence of positive eigenvalues for a class of subelliptic operators, Math. Ann., 304 (1996), 701-715, MR 97f:35155, Zbl 0847.35094.

[8] D. Guo, Inequalities for eigenvalues of polyharmonic operators, Acta Math. Appl. Sinica, 15(4) (1992), 451-459, CMP 1327 083, Zbl 0789.35120.

[9] G.N. Hile and M.H. Protter, Inequalities for eigenvalues of the Laplacian, Indiana Univ. Math. J., 29 (1980), 523-538, MR 82c:35052, Zbl 0454.35064.

[10] G.N. Hile and R.Z. Yeh, Inequalities for eigenvalues of the biharmonic operator, Pacific J. Math., 112 (1984), 115-133, MR 85k:35170, Zbl 0541.35059.

[11] J.J. Kohn, Boundaries of complex manifolds, Proc. Conf. on complex manifolds, Minneapolis, 1964, 81-94, MR 30 \#5334, Zbl 0166.36003.

[12] X. Luo and P. Niu, Eigenvalue problems for square sum operators consisting of vector fields, Math. Appl., 10(4) (1997), 101-104, MR 99c:35175, Zbl 0932.35155.

[13] _ On the eigenvalues of the operator sum of squares of vector fields, Chin. Bull. Sci., 43 (1998), 263. 
[14] , On the Dirichlet eigenvalue problem of the Folland-Stein operator in the Heisenberg group, Acta Math. Sci., 17(suppl.) (1997), 70-75.

[15] A. Menikoff and I. Sjöstrand, On the eigenvalues of a class of hypoelliptic operators, Math. Ann., 235 (1978), 55-85, MR 58 \#1735, Zbl 0375.35014.

[16] L.H. Payne, G. Polya and H.F.Weinberger, On the ratio of consecutive eigenvalues, J. Math. Phys., 35 (1956), 289-298, MR 18,905c, Zbl 0073.08203.

Received October 22, 1999 and revised March 29, 2002. This research was supported by the National Natural Science Foundation of China.

Department of Applied Mathematics

Northwestern Polytechnical University

Xi'An, ShaAnXi, 710072

People's Republic of China

E-mail address: taoer558@sohu.com

Department of Applied Mathematics

Northwestern Polytechnical University

XI'An, ShaAnXi, 710072

People's Republic of China 